\title{
CU\$TO CAPRINO CORTE 1.0: SOFTWARE DE CONTROLE DE CUSTOS PARA A CAPRINOCULTURA DE CORTE
}

\author{
CU\$TO CAPRINO CORTE 1.0: A cost control software for beef goat production
}

\author{
Marcos Aurélio Lopes, Romildo de Pinho Campello², Francisval de Melo Carvalho ${ }^{3}$, \\ Delmara de Cássia Fernandes Lopes ${ }^{4}$
}

\begin{abstract}
RESUMO
Realizou-se este trabalho com objetivo de desenvolver um software para auxiliar os técnicos e caprinocultores na determinação do custo de produção de caprinos de corte. O software CU\$TO CAPRINO CORTE 1.0 é composto de um plano de contas, no qual o usuário pode cadastrar todas as despesas e receitas referentes ao sistema de produção de cabras de corte. O software permite o cadastramento de todos os bens móveis e benfeitorias do sistema de produção, visando os cálculos de depreciação e remuneração do capital. O CU\$TO CAPRINO CORTE 1.0 calcula e apresenta ao usuário as seguintes variáveis: total das receitas, total dos custos operacionais, custo total, margem bruta, margem líquida, lucro, custo operacional e custo total por kg de carne, ponto de equilíbrio do sistema de produção, quantidade total de carne produzida, valor do patrimônio, remuneração do capital, lucratividade e rentabilidade. O software permite ao usuário diversas simulações envolvendo diversos parâmetros e variáveis, mostrando os pontos de estrangulamento e auxiliando o técnico e o pecuarista na determinação do custo de produção de caprinos de corte com precisão e considerável rapidez.
\end{abstract}

Termos para indexação: Custo de produção, gerenciamento, informática, sistema computacional.

\begin{abstract}
The objective of this work was to develop a software to help both technicians and raisers in determining the production cost for beef goat. The CU\$TO CAPRINO CORTE 1.0 software is made up of an account plan in which the user can input all the expenses and incomes concerning the beef goat production. The software allows to register all the movables and immovables of the production system aiming at the calculations of depreciation and remuneration of capital. The CU\$TO CAPRINO CORTE 1.0 calculates and presents to the user the following variables: total of the operational costs, total costs, total of the incomes, coarse margin, net margin, profit, operational cost and total cost per weight unit of meat, break even point of production system, total amount of the meat produced, value of the property and remuneration of capital. The software allows to the user a number of simulations involving several parameters and variables showing the bottleneck points and helping the technicians and raisers in the determination of beef goat production costs with precision and considerable quickness.
\end{abstract}

Index terms: Beef goat, computer science, management, production cost, software.

(Recebido para publicação em 3 de agosto de 2004 e aprovado em 11 de abril de 2005)

A agropecuária brasileira encontra-se em um momento que exige e propicia a geração, difusão e utilização de modernas técnicas de produção agropecuária e de administração da propriedade agrícola. Só assim, o agricultor terá instrumentos efetivos para auxiliá-lo no planejamento, implementação, direção e controle das atividades a serem executadas (ABCZ, 2001). De acordo com Ajimastro Júnior \& Paz (1998), é necessário introduzir novas tecnologias de suporte administrativo, rompendo com práticas tradicionais e possibilitando o surgimento de novas abordagens gerenciais.

No processo de informatização e modernização da pecuária, diversas são as aplicações e usos da informática, muitas dessas relatadas por Lopes (1997, 2002). Dentre essas, destaca-se o desenvolvimento de sistemas computacionais, visando ajudar caprinocultores e profissionais a tomarem uma decisão correta.

No Brasil, segundo Lopes (2002), o mercado de softwares agropecuários tem crescido. Em um levantamento realizado por esse pesquisador foi constatado um aumento de 54\% na oferta de softwares agropecuários em 1997, quando comparado com 1995 e 17\% em 1999, quando comparado com 1997. Analisando o Guia Agrosoft, o aumento foi de $24 \%$ em 2004. Do total de 212 softwares cadastrados, nenhum abordou o assunto custo de produção e análise de rentabilidade na caprinocultura.

\footnotetext{
${ }^{1}$ Professor do Departamento de Medicina Veterinária da Universidade Federal de Lavras/UFLA, DSc - Cx. P. 3037 - $37200-000$ - Lavras, MG malopes@ufla.br.

${ }^{2}$ Diretor da Geocamp - Geoprocessamento e Informática - Acadêmico do curso de Ciência da Computação da Universidade Brás Cubas romildo@geocampdigital.com

${ }^{3}$ Professor da Universidade Federal de Lavras/UFLA.

${ }^{4}$ Técnica em Contabilidade e Técnica em Processamento de Dados.
} 
Atualmente, os negócios agropecuários revestemse da mesma complexidade e dinâmica dos demais setores da economia, requerendo do produtor uma nova visão da gestão dos seus negócios, principalmente pela necessidade de abandonar a posição tradicional de fazendeiro para assumir o papel de empresário rural (YAMAGUCHI \& CARNEIRO, 1997). A necessidade de analisar economicamente a atividade é extremamente importante, pois, através dela, o produtor passa a conhecer com detalhes e a utilizar, de maneira inteligente e econômica, os fatores de produção (terra, trabalho e capital). A partir daí, localiza os pontos de estrangulamento para depois concentrar esforços gerenciais e tecnológicos, para obter sucesso na sua atividade e atingir os seus objetivos de maximização de lucros e minimização de custos (LOPES \& CARVALHO, 2002). Para administrar qualquer empresa, o primeiro passo é conhecer essa empresa e o mundo em que ela está inserida. Quanto mais conhecimentos da empresa, do seu funcionamento, e do ambiente em que ela está inserida tiver o administrador, maiores serão as chances dele tomar decisões acertadas. Para se conhecer bem um sistema de produção de cabras de corte necessário se faz conhecer, dentre outras coisas, o custo da carne produzido por ele.

Considerando a complexidade da estimativa do custo de produção da carne, que a maioria das fazendas tem seus proprietários como administradores e a inexistência de um software específico sobre o tema, resolveu-se realizar este trabalho, cujos objetivos foram: 1) desenvolver um software para auxiliar os técnicos e caprinocultores na determinação do custo de produção da caprinos de corte; 2) desenvolver uma ferramenta que possibilite ao técnico e ou produtor efetuar simulações em um sistema de produção de carne de caprinos; e 3) apresentar o sistema computacional Custo Caprino Corte 1.0, cuja finalidade é determinar o custo de produção de caprinos de corte.

O CU\$TO CAPRINO CORTE 1.0 foi desenvolvido utilizando a ferramenta Visual Basic. Os cálculos do custo de produção da caprinocultura de corte contemplaram as duas estruturas de custo de produção: Custo Total de Produção (clássica), que envolve o custo fixo e variável utilizada por Barros (1948) e Custo Operacional, proposta por Matsunaga et al. (1976) e adotada pelo Instituto de Economia Agrícola - IEA/SP (MATSUNAGA et al., 1976).

A metodologia utilizada nos cálculos da Margem Bruta, Margem Líquida e ponto de equilíbrio foi a adotada por Reis (1986). A depreciação foi calculada pelo método linear (HOFFMANN et al., 1981). A rentabilidade e a lucratividade foram calculadas conforme o preconizado por Matarazzo (1997). Os itens que compõem o Custo Operacional Efetivo de produção do caprinocultura de corte foram divididos em sete grupos: Mão-de-obra, Alimentação, Sanidade, Inseminação artificial, Compra de animais, Impostos e Despesas Diversas (LOPES et al., 1999).

O software desenvolvido deve ser utilizado sobre a plataforma Windows 95 ou superior, não atendendo às plataformas anteriores como o Windows 3.11. As rotinas foram desenvolvidas de forma conversacional, com acesso às diversas opções por meio de menus auto-explicativos, o que permite a operação do sistema por pessoas não especializadas em informática, mas apenas treinadas na operação de microcomputadores. Tal fato possibilita um maior uso do software no meio rural, onde a informática não é muito difundida (LOPES, 1997).

O menu principal, apresentado na forma de "fichas", contém as opções Lançamentos, Grupos, Relatórios e Gráficos, Configurações, e Créditos (Figura 1). Para selecionar uma opção o usuário deve posicionar o mouse e clicar sobre a opção desejada. Esse recurso facilita a operação do software, mostrando ao usuário todas as opções disponíveis.

Em Lançamentos estão disponíveis as opções Despesas, Produção, Inventário e Dados do mês. Selecionando uma das opções é disponibilizado um menu secundário, também na forma de "fichas", contendo as opções: Edição de dados e Visualização geral.

Selecionando Despesas, na opção Edição de dados, o usuário realiza o lançamento das despesas nos respectivos Grupos e Sub-grupos, preenchendo os devidos campos (Figura 1). A divisão das despesas em grupos permite ao usuário monitorar as despesas do sistema de produção de caprinos de corte, mês a mês, auxiliando o técnico e ou produtor em uma análise mais detalhada, objetivando encontrar possíveis pontos de estrangulamento. Na Ficha Visualização Geral tem-se uma visão panorâmica de todas as despesas já cadastradas.

Em Produção, assim como em Despesas, é disponibilizado um menu secundário, contendo as opções Edição de dados e Visualização geral. Em Edição de dados, o usuário realiza o cadastramento das receitas nos respectivos Grupos e Sub-grupos, preenchendo os devidos campos (Figura 2). Na Ficha Visualização Geral tem-se uma visão panorâmica de todas as produções já lançadas.

Ciênc. agrotec., Lavras, v. 29, n. 5, p. 1061-1068, set./out., 2005 


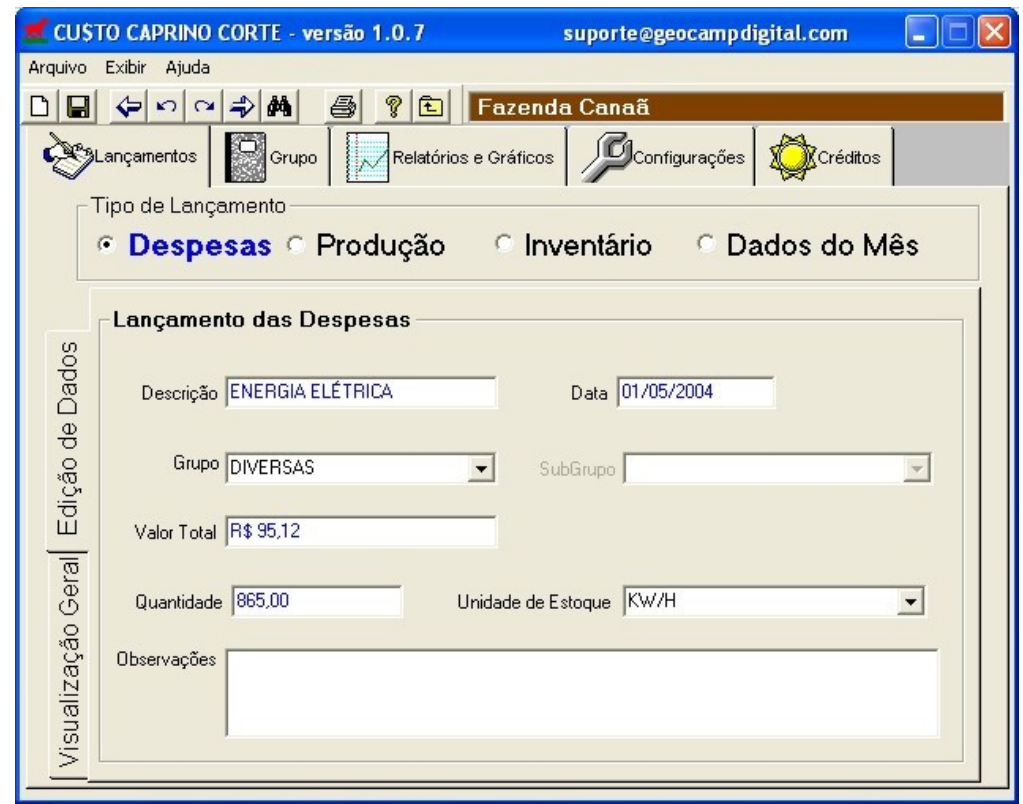

FIGURA 1 - Tela contendo a opção Despesas.

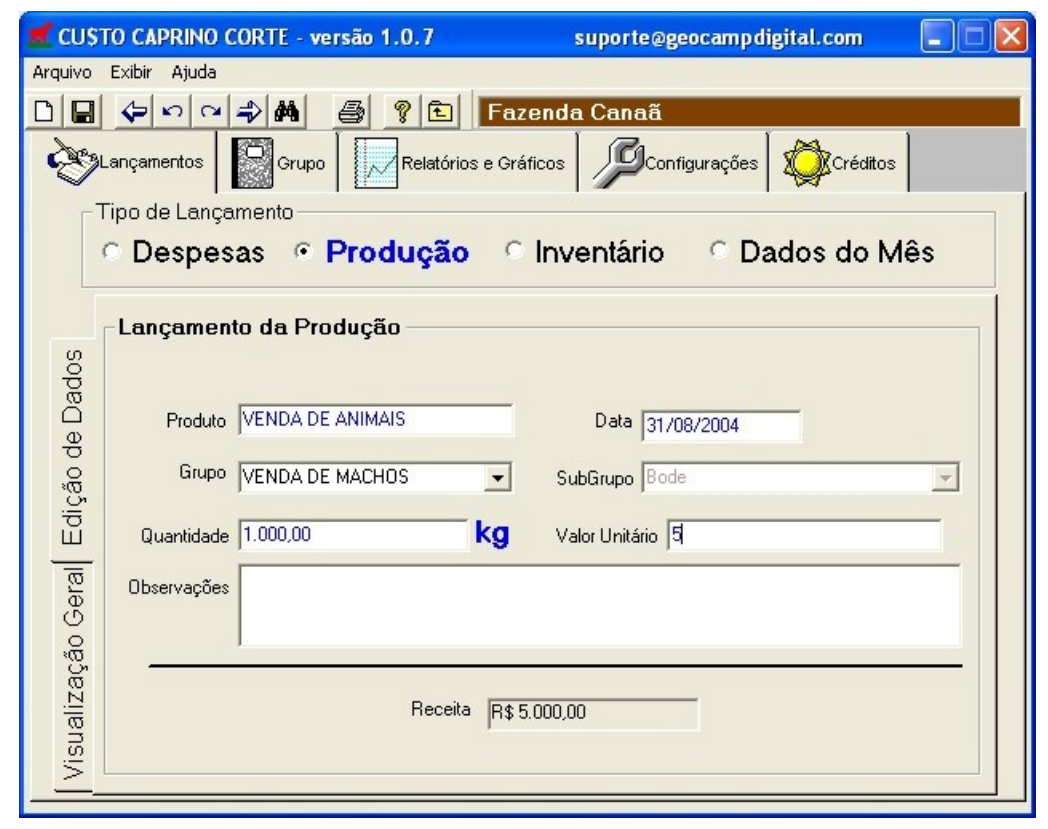

FIGURA 2 - Tela contendo a opção Produção. 
Em Inventário o usuário deve cadastrar todos os bens móveis e benfeitorias do sistema de produção de caprinos de corte, preenchendo os campos: Item, Data de aquisição, Grupo, Quantidade, Valor unitário, Vida útil e Valor unitário de sucata (Figura 3). Os demais campos (Valor da aquisição, Valor total de sucata, Depreciação mensal e Depreciação anual) são preenchidos automaticamente pelo CU\$TO CAPRINO CORTE 1.0. Cada descrição é classificada segundo um grupo básico do inventário, prédefinido pelo software. O preenchimento correto dessa "ficha", além de proporcionar maior precisão nos cálculos, dá ao produtor o valor real do patrimônio e do capital investido na atividade pecuária de corte (LOPES et al., 1999). Devem ser cadastrados apenas os bens utilizados na atividade. Caso um bem seja utilizado também em outra atividade da propriedade, deve ser atribuído, por meio de rateamento, um valor para a atividade caprinocultura de corte e esse valor deve ser o utilizado pelo CU\$TO CAPRINO CORTE 1.0.

O cadastro do inventário é um importante item, pois esses valores são utilizados pelo software para calcular a remuneração do capital investido, bem como as depreciações. Tal cadastro talvez seja a tarefa mais árdua realizada pelo usuário nesse processo do cálculo do custo da produção da caprinocultura de corte. Mas vale ressaltar que tal tarefa é realizada apenas uma vez por ano ou por ocasião da aquisição de um bem, e não mês a mês, como o cadastro das despesas, por exemplo.

Em Dados do Mês (Figura 4), assim como em Despesas, é disponibilizado um menu secundário, contendo as opções Edição de dados e Visualização geral. Em Edição de dados, o usuário deve também atribuir e registrar um valor para remuneração do empresário, valor do arrendamento da terra, taxa real de juros mensal, área do sistema de produção e valor da terra. Os valores da remuneração do empresário e da terra são utilizados para a determinação do custo de oportunidade do capital; a taxa real de juros, para remunerar o capital de giro e o capital investido; o valor da terra é utilizado na estimativa de rentabilidade da atividade.

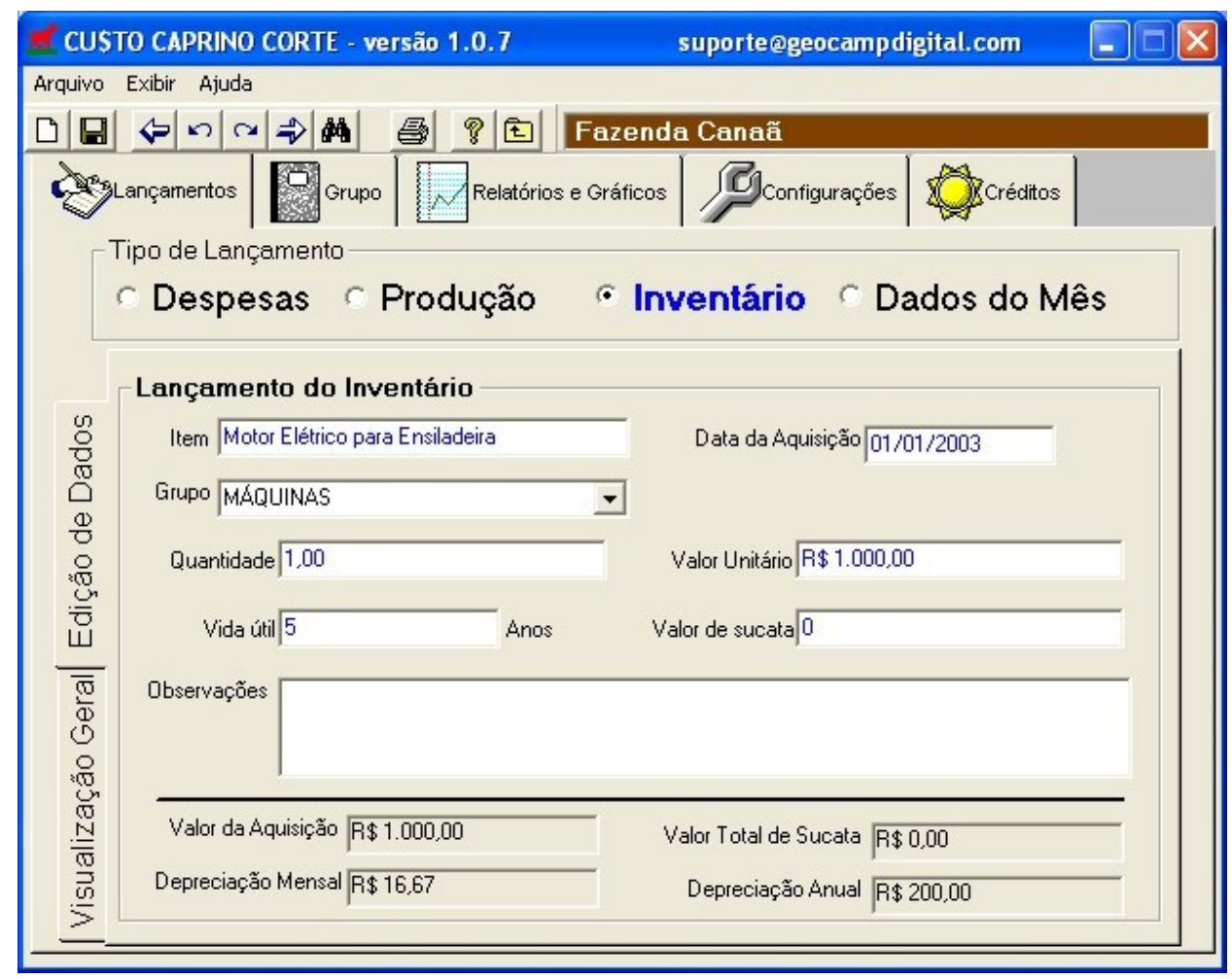

FIGURA 3 - Tela contendo a opção Inventário.

Ciênc. agrotec., Lavras, v. 29, n. 5, p. 1061-1068, set./out., 2005 


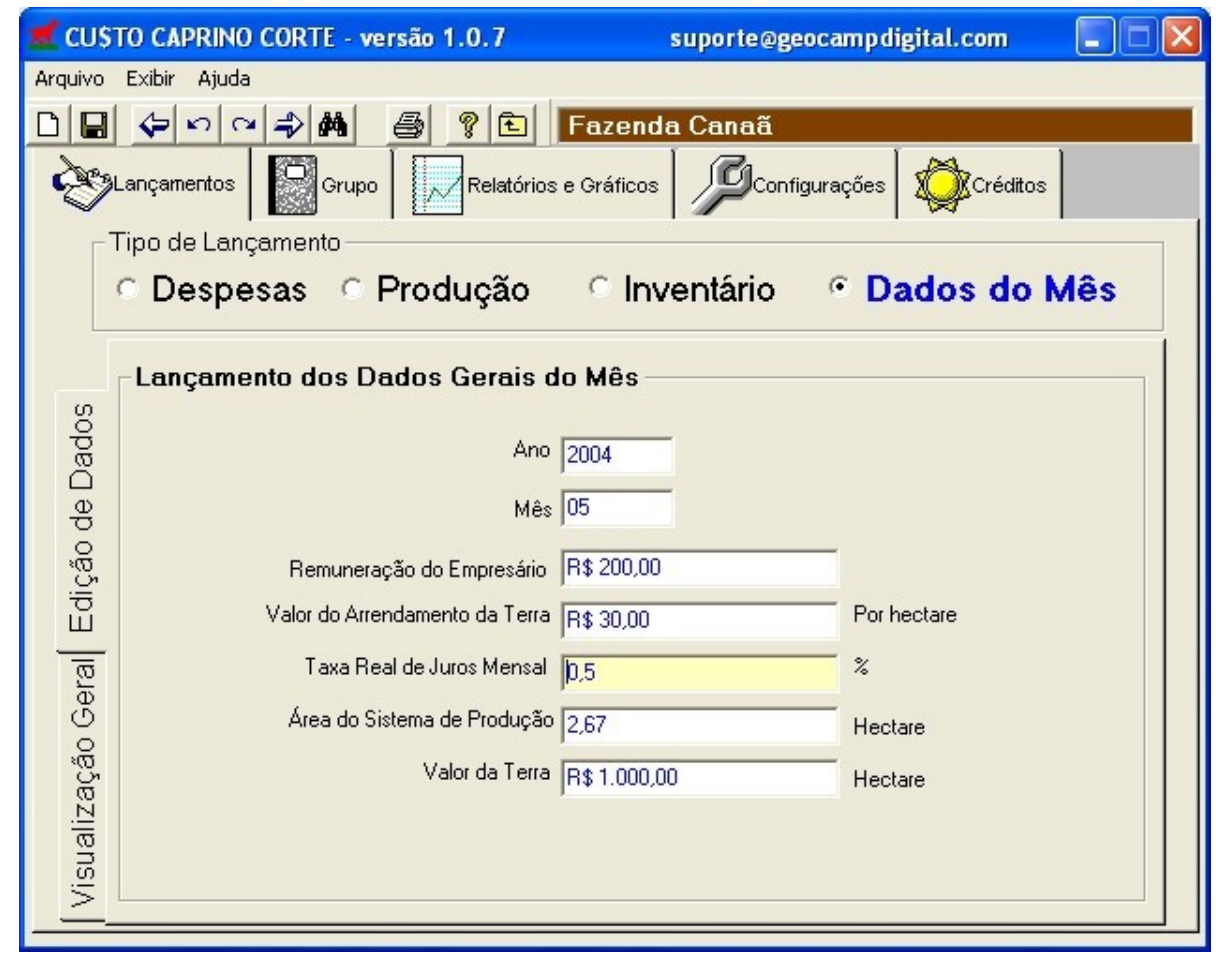

FIGURA 4 - Tela contendo a opção Dados do Mês.

Em Grupos estão disponíveis as opções Despesas, Produção e Inventário. O software CU\$TO CAPRINO CORTE 1.0 já traz alguns Grupos que servirão de base para a construção do plano de contas do sistema de produção. Para hierarquizar o projeto de administração da empresa rural, o usuário poderá criar sub-grupos. A título de exemplo, pode-se sub-dividir o grupo de despesas com Alimentação em vários sub-grupos, tais como: concentrado, mineral e volumoso. Torna-se importante salientar que o usuário é responsável pela criação do plano de contas, adaptando-o da forma que melhor lhe prouver. Assim, o software torna-se uma ferramenta flexível às necessidades diversas do processo de tomada de decisão de cada sistema de produção. Tal característica é de fundamental importância.

Em Relatórios e Gráficos estão disponíveis as opções Despesas, Produção e Receita, Inventário e Consolidação (Figura 5). O usuário, após indicar o período inicial e final, pode solicitar ao CU\$TO CAPRINO CORTE 1.0 diversos relatórios, instrumentos estes que lhe auxiliarão em uma análise mais detalhada do sistema de produção de caprinos de corte.

Na opção relatório de Consolidação é gerada toda a análise de rentabilidade do sistema de produção de caprinos de corte e alguns indicadores de desempenho técnico são mostrados ao usuário (Figura 6). Tais resultados permitem avaliar e orientar as decisões a serem tomadas pelo produtor e ou pelo técnico. Neste relatório são apresentados os seguintes resultados: receitas, custo operacional total, custo operacional efetivo, custo total, custos fixos, custos variáveis, margem bruta, margem líquida, resultado (lucro ou prejuízo), ponto de equilíbrio, produção total, lucratividade, rentabilidade, relação custo fixo/custo total, relação custo variável/custo total. Os seguintes custos médios (ou unitários), por kg, são calculados e podem ser observados no relatório de consolidação: custo operacional total, custo operacional efetivo, custo variável unitário e custo total. Também nesse relatório é mostrado o preço médio do $\mathrm{kg}$ de carne praticado pelo pecuarista durante o período estipulado para emissão do relatório. 


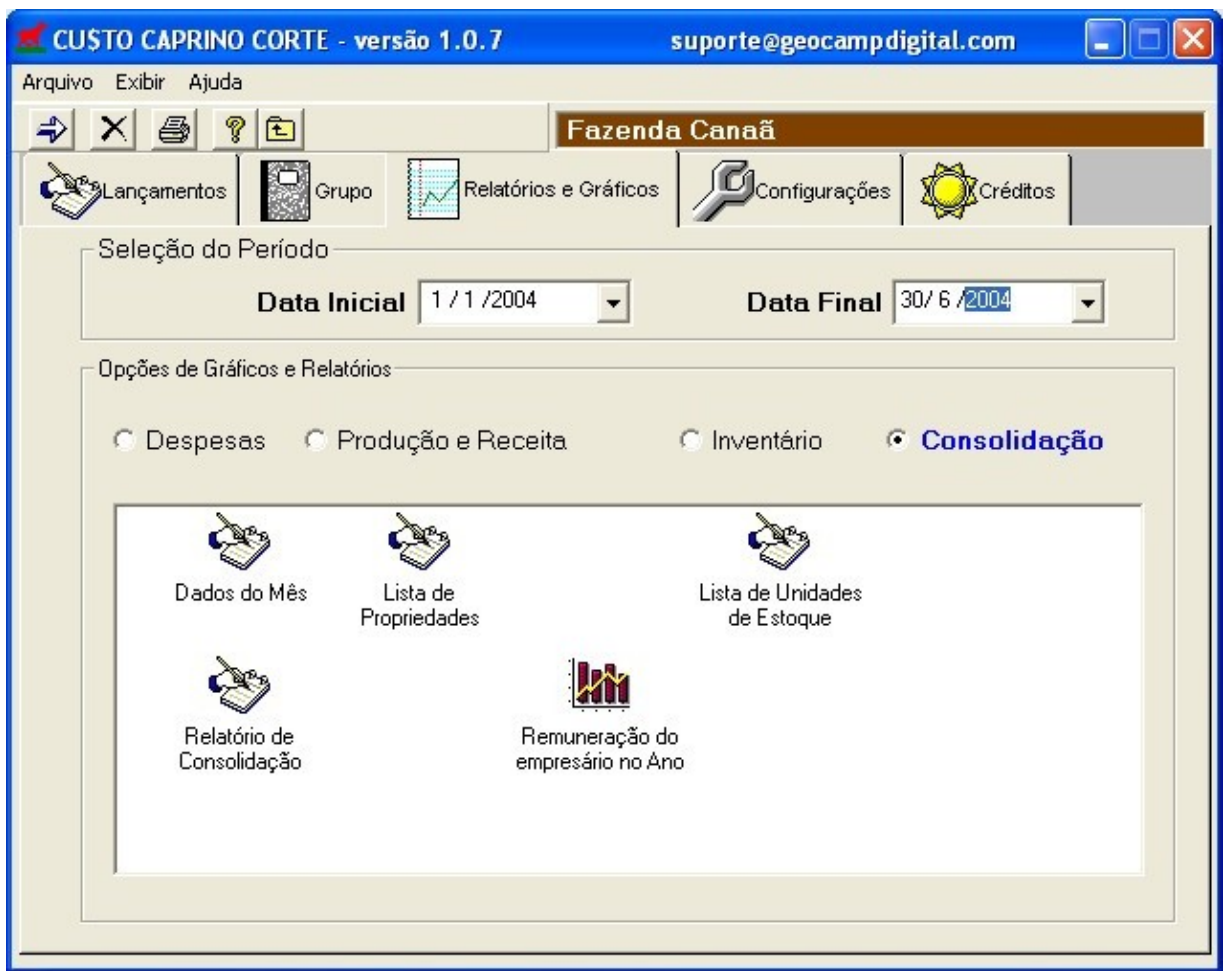

FIGURA 5 - Tela contendo a opção Relatórios e Gráficos - Consolidação.

Visando uma melhor e mais detalhada análise o CU\$TO CAPRINO CORTE 1.0 emite os diversos relatórios imprimindo-os diretamente em impressora, na tela do monitor e ou em arquivo. Esse recurso possibilita um melhor armazenamento da documentação gerada e aumenta as possibilidades de transmissão dos dados através de facsimile, fax modem e internet. O software possui diversos botões, visando uma maior facilidade na operação. Esses, uma vez acionados, permitem ao usuário localizar os dados já cadastrados, eliminar ou inserir novos dados.

Em configurações estão disponíveis as opções Propriedades, Unidades, Segurança e Habilitação. Selecionando Propriedades, o usuário tem uma visão geral de todas as empresas rurais cadastradas, podendo inserir novas fazendas e excluir as já cadastradas. Em Unidades, estão relacionadas algumas unidades de medidas comumente utilizadas. O usuário, assim como em Propriedades, possui botões que lhe permitem inserir e excluir unidades.
Segurança vem a ser uma ferramenta de extrema importância, pois permite que o usuário faça cópia de segurança (backup) e substitua os dados já cadastrados (restore); Habilitação constitui uma ferramenta para evitar instalações do CU\$TO CAPRINO CORTE 1.0 sem a devida autorização da Empresa desenvolvedora.

$\mathrm{O}$ software possui, inserido no help on line, um glossário de termos técnicos, opção importante para auxiliar o usuário na execução e entendimento da complexa análise de rentabilidade da atividade produção de caprinos de corte.

O sistema computacional desenvolvido é uma importante ferramenta que: auxilia o técnico e o pecuarista na determinação do custo de produção de caprinos de corte; fornece informações importantes para a tomada de decisões pelos pecuaristas; e permite ao usuário diversas simulações englobando diversos parâmetros e variáveis envolvidas em um sistema de produção de caprinos de corte. 


\section{Fazenda Canaã}

Relatório : CONSOLIDAÇÃO

Período: de 01/05/2001 à 31/08/2001

A - Receitas

B - Custo Operacional Total [ C + D ]

C - Custo Operacional Efetivo (despesas)

D - Custo com Depreciação

E - Custo Total [ F + L ]

F - Custos Fixos [ G+H+I+J+K ]

G - Remuneração da Terra

H - Remuneração sobre o Capital Investido

I - Remuneração do Empresário

$\mathrm{J}$ - Custo com Depreciação

$\mathrm{K}$ - Custo com Impostos

L - Custos Variáveis [ $\mathbf{M}+\mathbf{N}$ ]

$M$ - Custo Operacional Efetivo sem Impostos

N -Remuneração sobre o Capital de Giro
04/08/2004

Página: 1
R\$ 87.048,76
R\$ 69.997,27
$R \$ 68.971,27$
$R \$ 1.026,00$
R\$ 72.190,76
R\$ 2.730,58
R\$ 291,78
R\$ 852,00
$R \$ 360,00$
$R \$ 1.026,00$
$R \$ 200,80$

R\$ 69.460,18

RS 68.770,47

R\$ 689,71

R\$ $18.077,49$

RS $17.051,49$

R\$ 14.858,00

R\$ 42, 07

$\mathrm{R} \$ 42,70$

R\$ 44,03

R\$ 42,37

$\mathbf{R} \$ 48,65$

$1.639,44 \mathrm{~kg}$

$434,68 \mathrm{~kg}$

$1.204,76 \mathrm{~kg}$

$17,07 \%$

$13,01 \%$

$3,78 \%$

$96,22 \%$

$1,47 \%$

$98,53 \%$

GEOCAMP - 1147905902 - suporte@geocampdigital.com

FIGURA 6 - Tela contendo o Relatório de Consolidação. 


\section{REFERÊNCIAS BIBLIOGRÁFICAS}

ASSOCIAÇÃO BRASILEIRA DOS CRIADORES DE ZEBU. A pesquisa mostra o caminho de precocidade no gado zebu. Informativo ABCZ, Uberaba, n. 149, ago. 2001.

AJIMASTRO JÚNIOR, C.; PAZ, M. E. da. Identificação eletrônica. In: CONGRESSO BRASILEIRO DAS RAÇAS ZEBUÍNAS, A INTEGRAÇÃO DA CADEIA PRODUTIVA, 3., 1998, Uberaba, MG. Anais... Uberaba: ABCZ, 1998. p. 167-169.

BARROS, H. Economia agrária. Lisboa: Sá da Costa, 1948. $348 \mathrm{p}$.

HOFFMANN, R.; ENGLER, J. J. C.; SERRANO, O. Administração da empresa agrícola. 3. ed. São Paulo: Pioneira, $1981.325 \mathrm{p}$.

LOPES, M. A. Informática aplicada à bovinocultura. Jaboticabal: FUNEP, 1997. 82 p.

LOPES, M. A. Informática aplicada à bovinocultura leiteira. Lavras: UFLA, 2002. 130 p. Apostila do curso de especialização por tutoria à distância Bovinocultura leiteira: manejo, mercado e tecnologias.
LOPES, M. A.; CARVALHO, F. C. de. Custo de produção do gado de corte. Lavras: UFLA, 2002. 47 p. (Boletim agropecuário, 47).

LOPES, M. A.; JUNQUEIRA, L. V.; ZAMBALDE, A. L. Desenvolvimento de um sistema computacional para determinação do custo de produção do bovinocultura de corte. Revista Brasileira de Agroinformática, Viçosa, v. 2, n. 2, p. 105-116, 1999.

MATARAZZO, D. C. Análise financeira de balanços. 4. ed. São Paulo: Atlas, 1997. 463 p.

MATSUNAGA, M.; BEMELMANS, P. F.; TOLEDO, P. E. N. Metodologia de custo de produção utilizado pelo IEA. Agricultura em São Paulo, São Paulo, v. 23, n. 1, p. 123-139, 1976.

REIS, D. L. dos. Estudo técnico e econômico da propriedade rural. Informe Agropecuário, Belo Horizonte, v. 12 , n. 143 , p. $23-38,1986$.

YAMAGUCHI, L. C. T.; CARNEIRO, A. V. Aplicação de planilha eletrônica na análise técnica e econômica de unidades de produção de leite. In: CONGRESSO DA SOCIEDADE BRASILEIRA DE INFORMÁTICA APLICADA À AGROPECUÁRIA E À AGROINDÚSTRIA, 1., 1997, Belo Horizonte. Anais... Belo Horizonte: SBIAGRO, 1997. p. $95-99$.

Ciênc. agrotec., Lavras, v. 29, n. 5, p. 1061-1068, set./out., 2005 